\title{
APPLICATION of MODEL-INDEPENDENT ANALYSIS to PEP-II RINGS *
}

\author{
Y. Cai, J. Irwin, M. Sullivan, and Y.T. Yan, SLAC, Stanford, CA 94309, U.S.A.
}

\section{Abstract}

Model Independent Analysis (MIA), which employs statistical methods to reveal one-micron charge-induced centroid changes in pulse-by-pulse beam-position-monitor (BPM) measurements at the SLAC linac [1] has been extended to storage rings, and is here applied at PEP-II to analyze transverse motion. The beam is resonantly excited by a shaker in the horizontal and vertical directions and 2050 consecutive-turn measurements of all BPMs are buffered. Four high-precision linearly-independent orbits are determined. Local Green's functions specified by the local transfer matrix components R12, R34, R32, and R14 are extracted from these orbits and fitted with the model lattice using an SVD-enhanced method. The BPM gain and cross-plane coupling along with 1 normal- and 1 skewquadrupole strength are determined per single-view BPM.

\section{INTRODUCTION}

Verification and diagnosis of an accelerator optics is desirable because the accuracy of the constructed accelerator beamlines (compared to the the designed lattice) directly determines the accelerator performance. In this paper, we present a technique extended from previous studies [1] for verifying the linear model of an electron/positron storage ring. The central part of this technique is to vary the quads and skews, as well as the equivalent quads and skews due to sextupole feed-downs in the lattice model to fit the local measured Green's functions which, in the linear case, are specified by values of the transfer matrix components, $R_{12}, R_{14}, R_{32}$, and $R_{34}$. Note that the measurement errors due to Beam Position Monitor (BPM) offsets, gains, crossplane couplings, and pin-cushion distortions may also be taken into account by making BPM gains and cross-plane couplings fitting variables in calculation of local Green's functions. Taking PEP-II Low-Energy Ring (LER) as an example, in section 2 we describe the BPM buffer data acquisition and the extraction of independent linear orbits. In section 3 we review the local Green's functions. We also review how the BPM gains and cross-plane couplings along with the strengths of the the normal quad families and the suitable skews are introduced as variables for the fitting between measurement and the lattice model. In section 4, we present some typical results from SVD-enhanced fitting. A brief summary is then given in section 5 .

${ }^{*}$ Work supported by DOE contract DE-AC03-76SF00515.

\section{EXTRACTION OF INDEPENDENT LINEAR ORBITS}

\subsection{BPM buffer data acquisition and manamge- ment}

In the PEP-II LER, there are 319 BPMs of which 160 BPMS are single-view horizontal (X) BPMs, 146 BPMs are single-view vertical (Y) BPMs, and 13 BPMs are double-view (X,Y) BPMs. Therefore, one-turn BPM buffer data can offer a maximum of $173 \mathrm{X}$ data and $159 \mathrm{Y}$ data. Unlike linacs where there is often enough incoming jitter in the beam to measure and identify betatron modes; in the rings, the beam is resonantly excited by a shaker either in the the horizontal direction at the horizontal betatron tune or in the vertical direction at the vertical betatron tune to offset synchrotron radiation damping. (Note that the horizontal and vertical tunes are actually the two eigen tunes of a coupling ring.) We usually take 2050 turn data for each buffer with either a horizontal or a vertical excitation and store these data in two matrices, one for the $\mathrm{X}$ data which is a 2050-by-173 matrix and the other for the $Y$ data which is a 2050-by-159 matrix. There may be bad BPMs and so we would perform a Singular Value Decomposition (SVD) of each data matrix to identify uncorrelated columns of data and then eliminate the corresponding BPM data [1].

\subsection{Extraction of independent linear orbits}

Once the data from bad BPMs is excluded from the Xdata and the Y-data matrices (become smaller-column matrices), one then performs FFT on each column of the data. The 0-th FFT mode, which is a real number for each column, represents the self-consistent closed orbit while another standout FFT mode, which is a complex number for each column and corresponds to the resonance excitation frequency, represents the two degrees of freedom of the betatron motion. The cosine-like orbit is represented by the real part and the sine-like orbit is represented by the imaginary part while the square root of the square sum of two represents the betatron amplitude. Therefore, for each buffer data with either a horizontal excitation or vertical excitation, one can obtain two independent linear orbits. As to be described in the next section, in order to calculate the local Green's functions and the global invariants, one needs 4 independent linear (X and $\mathrm{Y}$ ) orbits. This can be extracted from a complete set of data which contains one buffer data (both $\mathrm{X}$ and $\mathrm{Y}$ matrices) from a horizontal excitation and the other buffer data from a vertical excitation. Shown in figure 4 are typical independent linear orbits and the corresponding betatron amplitudes. One can clearly see the local couplings from these orbits. 

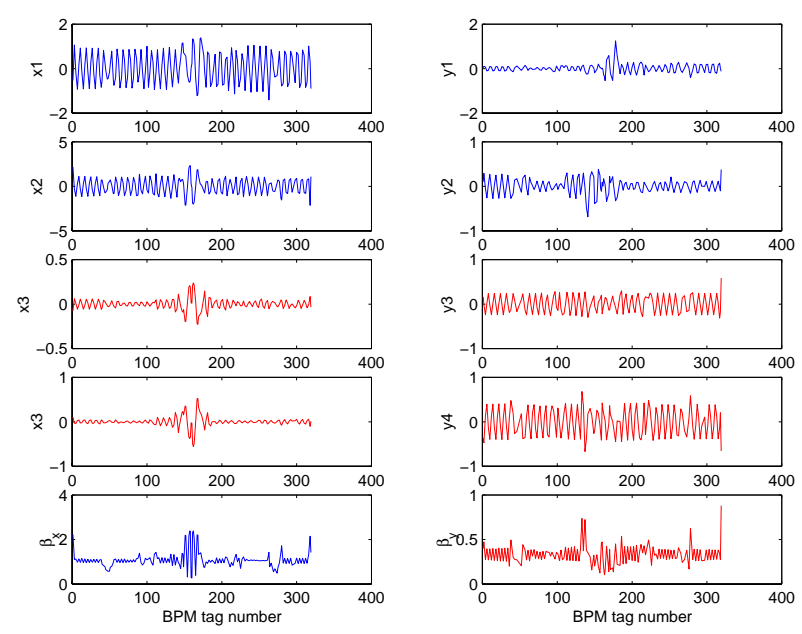

Figure 1: Four independent orbits extracted from PEP-II LER BPM buffer data. The first two orbits $(\mathrm{x} 1, \mathrm{y} 1)$ and $(\mathrm{x} 2, \mathrm{y} 2)$ are extracted from beam orbit excitation at the horizontal tune while the other two orbits (x3,y3) and (x4, y4) are from excitation at the vertical tune. The corresponding betatron amplitudes are shown at the bottom

\section{LINEAR GREEN'S FUNCTIONS}

Though x' and y' are not directly measured they exist. Given a complete set of 4 independent orbits obtained from one horizontal excitation and one vertical excitation, one can conceptually form a non-singular matrix at the $a^{\text {th }}$ or the $b^{\text {th }}$ BPM consisting of the phase-space coordinates: eg.

$$
Z^{a}=\left(\begin{array}{cccc}
x_{1}^{a} & x_{2}^{a} & x_{3}^{a} & x_{4}^{a} \\
x_{1}^{\prime a} & x_{2}^{\prime a} & x_{3}^{\prime a} & x_{4}^{\prime a} \\
y_{1}^{a} & y_{2}^{a} & y_{3}^{a} & y_{4}^{a} \\
y_{1}^{\prime a} & y_{2}^{\prime a} & y_{3}^{\prime a} & y_{4}^{\prime a}
\end{array}\right) .
$$

Applying the symplectic condition (damping is offset by the excitation to an equilibrium state), one obtains the invariants (constants around the ring) represented by an antisymmetric matrix $Q=Z^{b^{T}} J Z^{b}=Z^{a T} J Z^{a}$. This antisymmetric invariant matrix has, in general, 6 invariants. However, since the shaker is excited at the two eigen tunes, it can be shown that only the two non-coupled invariants $Q_{12}, Q_{34}$ are not 0 . The other 4 coupled invariants are all 0, i.e., $Q_{13}=Q_{14}=Q_{23}=Q_{24}=0$. Applying the symplectic property of the transfer map, $R^{a b^{T}} J R^{a b}=J$, and relationship between phase-space coordinates and measured orbits, $\left(x_{1}, y_{1}\right),\left(x_{2}, y_{2}\right),\left(x_{3}, y_{3}\right),\left(x_{4}, y_{4}\right)$ to the linear phase-space coordinates transfer relationship between $a$ and $b$ given by $Z^{b}=R^{a b} Z^{a}$, one obtains the following 4 equations [2]:

$$
\begin{aligned}
& \left(x_{1}^{a} x_{2}^{b}-x_{2}^{a} x_{1}^{b}\right) / Q_{12}+\left(x_{3}^{a} x_{4}^{b}-x_{4}^{a} x_{3}^{b}\right) / Q_{34}=R_{12}^{a b} . \\
& \left(x_{1}^{a} y_{2}^{b}-x_{2}^{a} y_{1}^{b}\right) / Q_{12}+\left(x_{3}^{a} y_{4}^{b}-x_{4}^{a} y_{3}^{b}\right) / Q_{34}=R_{32}^{a b} . \\
& \left(y_{1}^{a} x_{2}^{b}-y_{2}^{a} x_{1}^{b}\right) / Q_{12}+\left(y_{3}^{a} x_{4}^{b}-y_{4}^{a} x_{3}^{b}\right) / Q_{34}=R_{14}^{a b} . \\
& \left(y_{1}^{a} y_{2}^{b}-y_{2}^{a} y_{1}^{b}\right) / Q_{12}+\left(y_{3}^{a} y_{4}^{b}-y_{4}^{a} y_{3}^{b}\right) / Q_{34}=R_{34}^{a b} .
\end{aligned}
$$

The RHS's are always Green's function elements, $R_{12}, R_{32}, R_{14}, R_{34}$; the first index is 1 or 3 according to whether ' $b$ ' is horizontal or vertical, and the second index is 2 or 4 according to whether ' $a$ ' is horizontal or vertical. One or two or four of the above equations will apply depending on whether the two BPMs at ' $a$ ' and ' $b$ ' are both single-view or only one single-view or both double-view BPMs. Note that only measurements at ' $a$ ' and ' $b$ ' enter the equations; what happens at other BPM locations is actually irrelevant. The amplitude and orthogonality of the 4 modes being used enters through $Q_{12}$ and $Q_{34}$.

These linear Green's functions for all combinations of $(a, b)$ are not completely independent from each other. Since there are 4 measurements at each single-view BPM, 1 for each of the 4 orbits, one might expect each BPM to be involved in 4 independent equations. This is indeed true: there are 2 independent "normal" measurements and 2 independent "skew" measurements. For double-view BPMs one expects eight relationships. One can show that all Green's function elements may be expressed in terms of elements between neighbors and next-nearest neighbors.

Due to BPM gain errors and cross-couplings, Eqs. 14, have to be modified. Ideally, the BPM gains, $g_{x}$ 's, $g_{y}$ 's, and the cross-coupling multipliers, $\theta_{x y}$ 's and $\theta_{y x}$ 's can be introduced into the LHS of the Green's function equations if all BPMs are double-view BPMs. But this is not the case. Therefore, the equations are transformed into the measurement frame so that the LHS's of the equations are kept the same while the RHS's are modified such that $R_{12}, R_{32}, R_{14}$, and $R_{34}$ are replaced with $\mathcal{R}_{12}, \mathcal{R}_{32}, \mathcal{R}_{14}$, and $\mathcal{R}_{34}$, where [2]

$$
\begin{aligned}
& \mathcal{R}_{12}=g_{x}^{b} R_{12} g_{x}^{a}+g_{x}^{b} R_{14} \theta_{x y}^{a}+\theta_{x y}^{b} R_{32} g_{x}^{a}+\theta_{x y}^{b} R_{34} \theta_{x y}^{a}, \\
& \mathcal{R}_{32}=g_{y}^{b} R_{32} g_{x}^{a}+g_{y}^{b} R_{34} \theta_{x y}^{a}+\theta_{y x}^{b} R_{12} g_{x}^{a}+\theta_{y x}^{b} R_{14} \theta_{x y}^{a}, \\
& \mathcal{R}_{14}=g_{x}^{b} R_{14} g_{y}^{a}+g_{x}^{b} R_{12} \theta_{y x}^{a}+\theta_{x y}^{b} R_{34} g_{y}^{a}+\theta_{x y}^{b} R_{32} \theta_{y x}^{a}, \\
& \mathcal{R}_{34}=g_{y}^{b} R_{34} g_{y}^{a}+g_{y}^{b} R_{32} \theta_{y x}^{a}+\theta_{y x}^{b} R_{14} g_{y}^{a}+\theta_{y x}^{b} R_{12} \theta_{y a}^{a} .
\end{aligned}
$$

\section{RESULTS FROM PEP-II LER FITTING}

The LHS's of the modified Eqs. 1-4 can be directly calculated with the 4 independent orbits while the RHS can also be calculated if all $g_{x}$ 's, $g_{y}$ 's, $\theta_{y x}$ 's $\theta_{x y}$ 's are known and all $R_{12}, R_{32}, R_{14}$, and $R_{34}$ are calculated from the lattice model. Considering as variables all $g_{x}$ 's, $g_{y}$ 's, $\theta_{y x}$ 's $\theta_{x y}$ 's and the strengths of normal quad families and quad skews as well as the feed-downs of the sextupoles, one can iteratively achieve the best fit to the modified Eqs, 1-4. This yields the BPM errors and the difference between the lattice model and the built accelerator. We use an SVD enhanced method for the the fitting. First we obtain a linear derivative matrix such that the linearized difference (represented by a vector $\vec{\Delta}$ ) between the LHS and RHS of all the modified Green's function equations in each iteration of the fitting is given by $\vec{\Delta}=M \vec{\xi}$, where $\vec{\xi}$ is the increment of the variables to be determined through SVD analysis at each iteration. 

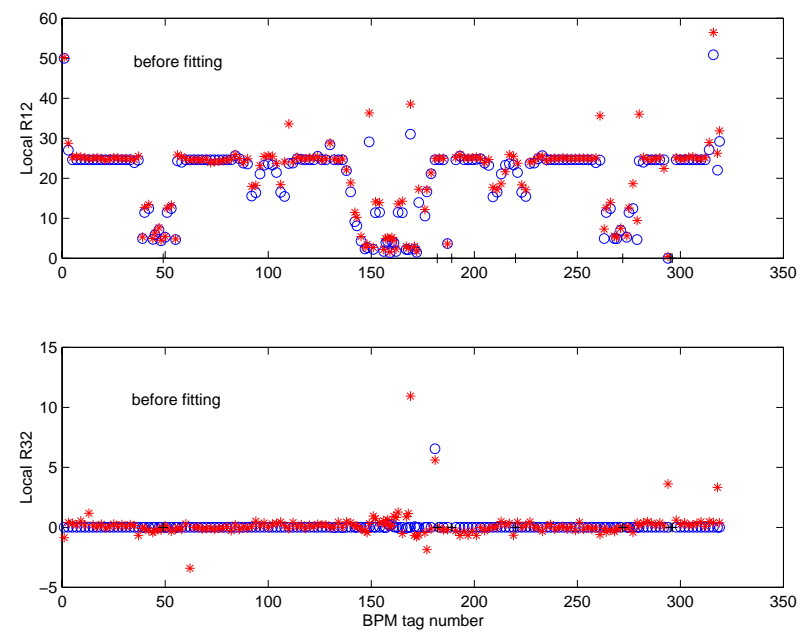

Figure 2: Comparison of local Greens' functions, $R_{12}$ and $R_{32}$, between measurement and that calculated from lattice model before fitting. The red stars are from measurement while the blue circles are from the lattice model.

Comparison of the Green's functions, $R_{12}$ 's and $R_{32}$ ' for adjacent BPMs of the entire ring are shown in Figure 2 before fitting and in Figure 3 after fitting for a typical PEPII LER case. The 'star' symbols represent the calculated values from LHS using the 4 independent orbits extracted from the BPM buffer data with a resolution enhancement by a factor of about $\sqrt{2000}$ while the 'circle' symbols represent the calculated values from the RHS of the modified Green's function equations. The residuals after fitting are much reduced Fitted values of BPM gains and cross-plane couplings and deviations between model and measurement for the magnet strengths are shown in Figure 4. The large gain errors (difference from 1) and the large cross-plane couplings (difference from 0 ) are errors from malfunctioning BPMs not identified before the fitting since the bad BPM data have been excluded. There are a few large magnet strength differences between measurement and lattice model. They are mostly those quadrupoles used for changing the tunes of the machine.

\section{SUMMARY}

We have extended MIA from the study of linacs to storage rings by fitting the local Green's functions between measurement and lattice model. The fitting results can identify malfunctioning BPMs and large magnet strength differences between the machine and the lattice model. However, for fine tuning of the machine, we still need some more effort to improve this technique in terms of (a) better choice of fitting variables at the right phase (right location), (b) more precise calculation of local Green's functions from the lattice model (such as modeling the solenoid more accurately). and (c) further improvement of the measurement resolution from our achieved factor of about $\sqrt{2000}$.
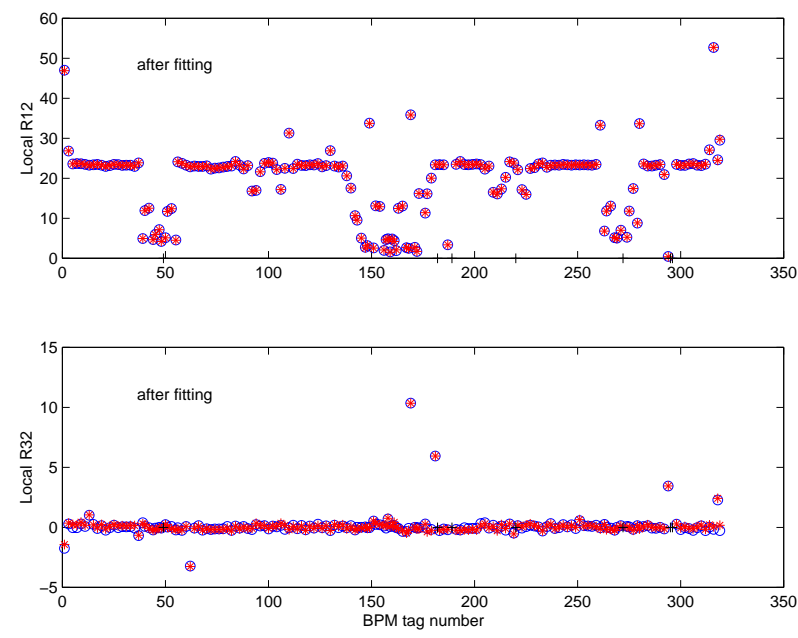

Figure 3: Comparison of local Greens' functions, $R_{12}$ and $R_{32}$, between measurement and that calculated from lattice model after fitting. The fitting variables are all BPM gains and cross-plane couplings and the strengths of the quad families and quad skews and sextupole feed-downs. The red stars are from measurement while the blue circles are from the lattice model.
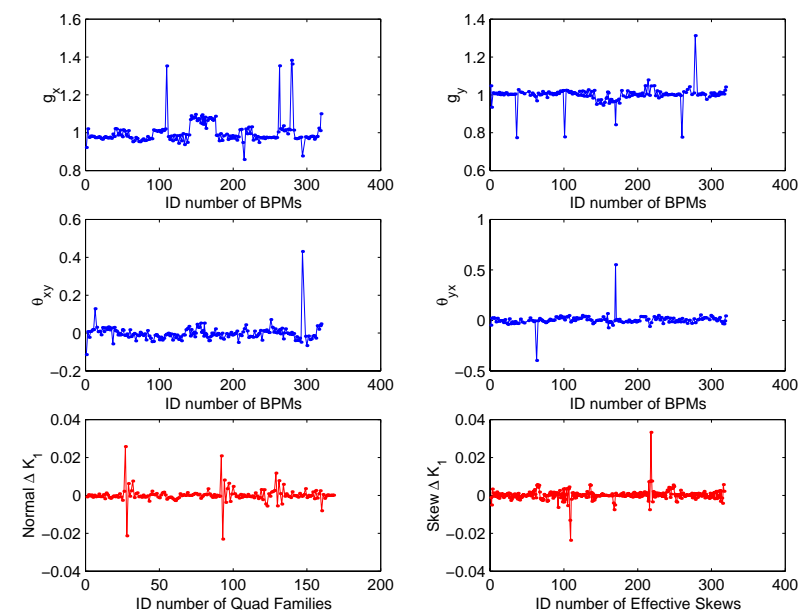

Figure 4: The BPM gains (the top plots) and the BPM cross-plane couplings (the middle plots) and the difference of the integrated magnet strength of the quad families and quad skews obtained from fitting for the local Green's functions between measurement and the lattice model. Note that bad BPM data have been excluded from fitting

\section{ACKNOWLEDGEMENT}

We thank T. Himel for help and J. Seeman and U. Wienands for support in PEP-II data acquisition.

\section{REFERENCES}

[1] J. Irwin, C.X. Wang, Y.T. Yan, et. al., "Model-Independent Beam Dynamics Analysis," Phys. Rev. Lett. 82, 1684 (1999).

[2] J. Irwin, and Y.T. Yan, "Beamline model verification using model-independent analysis” SLAC-PUB-8515 (2000). 